\title{
LABORATÓRIOS REMOTOS E VIRTUAIS NO BRASIL COM FOCO NO ENSINO: UMA REVISÃO SISTEMÁTICA DA LITERATURA
}

\author{
Ana Lúcia da Silva Beraldo, ICT/Unifesp, ana.beraldo@unifesp.br \\ https://orcid.org/0000-0003-4985-1888 \\ Tiago de Oliveira, ICT/Unifesp, tiago.oliveira@unifesp.br \\ https://orcid.org/0000-0002-3676-5967 \\ Denise Stringhini, ICT/Unifesp, dstringhini@unifesp.br \\ https://orcid.org/0000-0002-9729-4302
}

\begin{abstract}
Resumo. Esta revisão sistemática foi realizada para se ter uma visão geral do estado da arte sobre o uso de laboratórios virtuais e remotos dedicados ao ensino no Brasil. Buscou-se identificar quais são as principais aplicabilidades, vantagens, desvantagens, e verificar a importância do uso destes laboratórios para o ensino. Os resultados mostram que a maioria dos laboratórios remotos ou virtuais são aplicados na área de exatas e nos artigos pouco tem-se explorado sobre a eficácia da implantação do projeto no aprendizado do aluno, notou-se que a maioria dos artigos apresenta o desenvolvimento técnico do laboratório, fazendo avaliações com os alunos sobre a aceitação e usabilidade do sistema, sem apresentar estudos relacionados aos processos de ensino e de aprendizagem nesse ambiente educacional.
\end{abstract}

Palavras-chave: laboratório remoto e virtual, ensino, aprendizagem, revisão sistemática

\section{REMOTE AND VIRTUAL LABORATORIES IN BRASIL WITH FOCUS ON TEACHING: A SYSTEMATIC REVIEW OF LITERATURE}

\begin{abstract}
This systematic review was conducted to provide a state-of-the-art overview of the use of virtual and remote teaching laboratories in Brazil. We seek to identify the main applicabilities, advantages, disadvantages, and to verify the importance of using these laboratories to teaching. The results show that most remote or virtual laboratories are applied in the exact area. In the articles, little has been explored about the effectiveness of the project implementation in student learning. We noticed that most articles present the technical development of the laboratory, making assessments with students about the acceptance and usability of the system, without giving studies related to the teaching and learning processes in this educational environment.
\end{abstract}

Keywords: remote and virtual laboratory, teaching, education, systematic review

\section{Introdução}

O uso de laboratórios de ensino para a realização de práticas experimentais é essencial no processo de aprendizagem dos alunos. É fundamental que as instituições de ensino disponibilizem laboratórios práticos que atendam as demandas de seus cursos. Na última década, pôde-se perceber o aumento na implantação de laboratórios virtuais e remotos para suprir algumas lacunas. A implantação de laboratórios deste tipo, como poderemos ver nos próximos parágrafos é dada principalmente por falta de espaço físico e recursos financeiros para aquisição de equipamentos de alto custo.

O laboratório virtual possibilita atividades simultâneas, pois a sua característica principal é a simulação das atividades. Sendo assim, o aluno pode acessar o laboratório quantas vezes quiser para realizar os experimentos. De acordo com (GUAITA; GONÇALVES, 2014) "Laboratórios virtuais simulam funções essenciais que estejam relacionadas em um determinado experimento, neste caso a condição física da atividade é substituida por um modelo computacional". E, de acordo com (AMARAL et al., 2011) o uso do laboratório remoto "poderá contribuir para auxiliar as intervenções do professor e favorecer a autonomia dos alunos, estimulando-os na construção de conhecimentos significativos", fato que possibilita ao aluno ser o protagonista no processo de aprendizagem.

Já os laboratórios remotos, de acordo com Souza, Oliveira e Santos (2001) são laboratórios com equipamentos reais os quais podem ser acessados e controlados via internet. 
Neste tipo de laboratório, a instituição disponibiliza o equipamento para o uso remoto, então o aluno faz o agendamento de um período de tempo para realizar o acesso. É importante considerar que é essencial que exista comunicação com a rede da instituição, internet e energia elétrica para que o experimento aconteça de fato, se um destes itens falha não é possível realizar o experimento.

Devido à pandemia de COVID-19 no ano de 2020, os estabelecimentos de ensino tiveram que fechar suas portas e tiveram que oferecer aulas de forma online, síncronas ou assíncronas e "o uso de ferramentas virtuais tornou-se uma alternativa promissora para promover a continuidade das atividades educacionais" (DA COSTA NETO; DA SILVA COSTA, 2020) e desde então "as TICs e a internet têm sido cada vez mais utilizadas pelas instituições de ensino superior para suprir essa ausência nas salas de aulas" (GOMES, 2020) .

As disciplinas práticas foram duramente impactadas com estas restrições e uma alternativa para continuar o ensino e mitigar os prejuízos educacionais foi utilizar laboratórios remotos ou virtuais no ensino. Sendo assim, aquilo que outrora era implantado devido majoritariamente à falta de espaço físico e de recursos financeiros, tem se tornado uma alternativa possível durante a pandemia, possibilitando o exercício da prática respeitando-se $\mathrm{o}$ distanciamento social imposto pela atual pandemia vivenciada.

Devido à importância e utilidade dos laboratórios remotos e virtuais em processos de ensino e de aprendizagem, esta revisão sistemática identificou diversas soluções de laboratórios remotos e virtuais aplicados desde o ensino fundamental até o nível superior em todo o Brasil e tenta responder à questão: "Como ocorre o uso de laboratórios remotos e virtuais dedicados no processo de ensino no Brasil?".

O Ambiente Virtual de Aprendizagem, AVA, tem sido utilizado amplamente tanto para o ensino a distância quanto para o apoio ao ensino presencial. O AVA, de acordo com Frozza at al. (2011), é um local em que ocorrem interações cognitivo-sociais acerca de um assunto de estudo e não somente um local em que se disponibilizam conteúdos pedagógicos. Conforme Brito et al. (2013) nos AVAs podem ser incorporadas ferramentas como o bate-papo, os fóruns, wikis (que permitem que os participantes adicionem e editem um conteúdo colaborativamente). Os laboratórios remotos e virtuais podem estar incorporados aos AVAs ou ser disponibilizados diretamente em uma página desenvolvida para acesso ao laboratório.

Uma das principais motivações para o crescimento de tais laboratórios são os custos elevados com a implantação de laboratórios convencionais conforme observado por Gomes at al. (2009). Para um laboratório apoiar um curso presencial é necessário espaço físico, computadores e equipamentos específicos, sem contar o pessoal de apoio, entre outros. Também é citado por Ali at al. (2012) que o alto custo dos equipamentos para montagem de laboratórios dentre outros quesitos cria um gargalo significativo ao processo educacional.

Duarte Medina at al. (2004), que propuseram a criação de um laboratório virtual de redes de computadores em sua instituição, apontaram as seguintes dificuldades para se ter um laboratório tradicional: ausência de recursos financeiros, periculosidade dos experimentos e tempo de acesso restrito devido agenda do laboratório.

De Freitas at al. (2004), que propuseram um laboratório remoto para ensino de automação, indicaram algumas das vantagens do uso dos laboratórios remotos: ausência da necessidade de técnicos acompanhando o experimento, maior disponibilidade do experimento e rastreabilidade dos resultados.

A solução de uso de laboratórios remotos ou virtuais traz alguns benefícios aos usuários como maior tempo de uso dos equipamentos, menos danos físicos aos equipamentos devido à má utilização e, conforme verificado por Persiano at al. (2007), o ensino remoto com experimentos reais permite superar barreiras fornecendo flexibilidade no ensino. Os autores Rajasenkhar at al. (2008) em seu artigo, relatam que laboratórios remotos oferecem maior acessibilidade e potencial de abrir mais oportunidades para se aprender.

É importante oferecer o ensino prático aos cursos de computação e engenharias, proporcionando vivência real daquilo que se aprende. Neste contexto, conforme descrito por Indrusiak, Glesner e Reis (2007), a flexibilidade de acesso a kits de circuitos integrados digitais permite que os alunos configurem o laboratório remoto como um protótipo do sistema que eles estão projetando permitindo interação nas suas atividades, favorecendo, com isso, o aprendizado. 
E por fim, Zubia (2004), vislumbrou outras vantagens importantes dos laboratórios remotos como: organização do tempo de forma mais eficiente, apoio ao estudo independente, acesso de pessoas com deficiências físicas a equipamentos sofisticados a partir de sua casa e também a aproximação da tecnologia a países de terceiro mundo.

Descrita a relevância dos laboratórios remotos e virtuais para o ensino, a seção 2 abordará os objetivos e a metodologia empregada na realização deste trabalho; a seção 3 traz os resultados encontrados e, por fim, a seção 4 apresenta as considerações finais.

\section{Objetivos da pesquisa e metodologia de condução do trabalho}

A Revisão Sistemática "consiste em uma forma de síntese dos resultados de pesquisas relacionados com um problema específico" (GALVÃO; SWADA; TREVIZAN, 2009).

Algumas das características da revisão sistemática, indicadas por Kitchenham e Charters (2007) são: a definição da questão de pesquisa e os métodos que serão utilizados para conduzir a revisão sistemática, documentação da estratégia realizada na pesquisa para que a mesma possa ser replicada, definição de critérios de avaliação para inclusão e exclusão dos estudos, dentre outros. Tais critérios são utilizados, de acordo com Galvão, Swada e Trevizan (2009), para se evitar o viés no resultado da pesquisa científica proporcionando rigor em todas as fases do trabalho, de forma que possa ser reproduzida por outro autor e que se encontre os mesmos resultados. Todos estes processos são seguidos para que ao término se obtenha exatidão nos dados obtidos.

A metodologia adotada para esta revisão sistemática é a descrita pelos autores Ferenhof e Fernandes (2016), que propõe o método Systematic Search Flow - SSF, composto por quatro fases: protocolo, análise dos dados, síntese e a escrita do artigo baseando-se nas informações que foram consolidadas na fase de síntese e são respondidas as questões de pesquisa.

Nas próximas subseções são evidenciadas as questões de pesquisa, define-se a String de busca, descrevem-se as técnicas que são utilizadas para avaliar os artigos no processo de inclusão e exclusão na revisão sistemática e apresenta-se a matriz de conhecimento que traz as questões utilizadas para realizar a extração dos dados.

\subsection{Objetivos da pesquisa}

O objetivo deste trabalho é realizar uma compilação de estudos relacionados ao uso de laboratórios remotos e virtuais e sua aplicabilidade no processo de ensino-aprendizagem dos alunos. Além disso, os resultados obtidos da revisão sistemática realizada visam situar os educadores do que vem sendo pesquisado no Brasil e como vem sendo trabalhada a questão de adequação do laboratório remoto/virtual como ferramenta pedagógica e sua relação com o processo de aprendizagem do aluno.

Tema: Laboratórios remotos e virtuais com foco no ensino.

Objetivo: O principal objetivo desta revisão sistemática é fornecer uma visão geral dos trabalhos que tratam de laboratórios virtuais ou remotos e que descrevem a sua aplicação no processo de ensino no Brasil.

Questão principal: Como ocorre o uso de laboratórios remotos e virtuais relacionados ao ensino no Brasil?

Questões de pesquisa baseadas na questão principal e no objetivo da revisão:

QP1: Quais são as principais aplicabilidades e a importância do uso dos laboratórios virtuais e remotos?

QP2: Quais são as estratégias e métodos que têm sido adotados para medir a eficácia dessa abordagem no ensino?

QP3: Quais são as principais vantagens e desvantagens e problemas que vem sendo reportados na adoção dessa abordagem?

\subsection{Definição da String de busca e das bases de dados}


A estratégia de busca está separada em 3 partes: definição das palavras-chave, definição da String de busca e definição das bases de dados.

As palavras-chave definidas para o trabalho são:

- Laboratório Remoto, Laboratório Virtual, Laboratório Online

- Educação, Aprendizado, Ensino, Treinamento

A String de busca foi construída combinando-se as palavras-chave e utilizando os operadores lógicos "OR" e "AND":

(("laboratório remoto" OR "laboratório virtual” OR "laboratório online") AND ("educação" OR "aprendizado" OR "ensino" OR "treinamento"))

No entanto, quando a pesquisa, em alguma das bases de dados não gerou nenhum resultado, foi realizada nova busca utilizando só os termos ("laboratório remoto") e ("laboratório virtual").

As bases de dados selecionadas para este trabalho são as que tem alta relevância para o tema em estudo: Periódicos CAPES, Revista Brasileira de Educação, Revista Novas Tecnologias na Educação, Revista Brasileira de Informática na Educação, Simpósio Brasileiro de Informática na Educação, Workshop de Informática na Escola, Workshops do Congresso Brasileiro de Informática na Educação, Informática na Educação: teoria \& prática, Revista Brasileira de Ensino de Ciência e Tecnologia e Google Acadêmico.

Esta revisão sistemática buscou, nas bases de dados, conteúdo que tivesse relação com laboratórios remotos e virtuais que foram efetivamente aplicados ao ensino de forma geral no Brasil sem limitação por data.

\subsection{Matriz do conhecimento}

De acordo com Ferenhof e Fernandes (2016), a matriz de conhecimento é formada pelas questões que se deseja responder quando se faz uma revisão sistemática. No entanto, nem todas as questões podem ser respondidas em todos os artigos.

As questões desta revisão sistemática foram direcionadas ao contexto de laboratórios remotos ou virtuais. A matriz de conhecimento, apresentada na Figura 1 traz as questões que se buscava esclarecer.

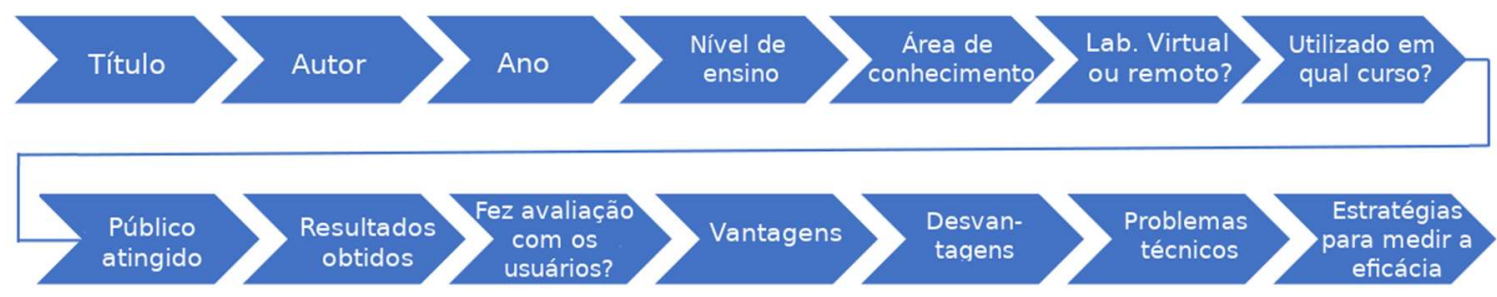

Figura 1 - Matriz de conhecimento desta Revisão Sistemática

\subsection{Critérios de seleção dos artigos}

A inclusão de critérios para a seleção dos artigos ajuda a filtrar os documentos relevantes daqueles que não tem ligação com o tema proposto. A Tabela 1 apresenta os critérios de inclusão e exclusão utilizados nesta revisão sistemática.

Tabela 1 - Critérios de inclusão e exclusão

\section{Critérios de INCLUSÃO}

Artigos que descrevam a utilização de laboratório remoto ou virtual e que tenham sido aplicados no ensino.

Artigos publicados em conferências, periódicos ou revistas científicas.

Artigos em que o projeto tenha sido aplicado no Brasil.

\section{Critérios de EXCLUSÃO}

Artigos que descrevam apenas a parte técnica de laboratório remoto ou virtual não apresentando resultados e a utilização para o ensino.

Artigos que não estejam relacionados com as questões de pesquisa.

Artigos em que o projeto não foi aplicado no Brasil. 
Artigos que estiverem escritos no idioma Artigos escritos em outros idiomas além de português ou inglês. português ou inglês.

\subsection{Obtenção dos artigos}

O primeiro passo foi realizar a busca nas bases de dados selecionadas utilizando a String de busca definida na seção 2.2. Os últimos acessos às bases de dados foram realizados na data de 04 de abril de 2021 e os resultados estão listados na Tabela 2. Devemos registrar que na busca realizada no Google Acadêmico foram verificados os 100 primeiros resultados retornados com o uso da String de busca.

Tabela 2 - Resultados obtidos nas bases de dados selecionadas

\begin{tabular}{lc} 
Base Bibliográfica & $\begin{array}{c}\text { Número de } \\
\text { artigos }\end{array}$ \\
\hline Periódicos CAPES & 34 \\
\hline Revista Brasileira de Educação & 2 \\
\hline Revista Novas Tecnologias na Educação & 9 \\
\hline Revista Brasileira de Informática na Educação & 5 \\
\hline Simpósio Brasileiro de Informática Na Educação & 18 \\
\hline Workshop de Informática na Escola & 5 \\
\hline Workshops do Congresso Brasileiro de Informática na Educação & 7 \\
\hline Informática na Educação: teoria \& prática & 2 \\
\hline Revista Brasileira de Ensino de Ciência e Tecnologia & 5 \\
\hline Google Acadêmico & 100 \\
\hline Total & $\mathbf{1 8 7}$ \\
\hline
\end{tabular}

Todos os 187 artigos foram analisados inicialmente pela leitura do título, abstract e foi verificado se atendiam à questão principal de pesquisa que é de ter sido utilizado para o ensino, nesta seleção já foram aplicados os critérios de inclusão e exclusão. Após esta filtragem e removendo as duplicidades sobraram 35 artigos que descrevem especificamente a implantação de um laboratório do tipo virtual ou remoto que foi utilizado para o ensino. Estes artigos foram lidos na íntegra e foi realizada a extração dos dados que permitiram responder as questões de pesquisa propostas. As referências completas dos 35 artigos utilizados nesta revisão sistemática são listadas no link: https://bit.ly/2NKHufo

$\mathrm{Na}$ listagem de referências bibliográficas consta, para cada artigo, um identificador [ID] o qual será utilizado na próxima seção para citar os artigos identificados durante a extração dos dados desta revisão sistemática.

\section{Resultados e discussão}

Os artigos selecionados neste trabalho foram publicados no período de 2004 a 2020, porém não havia critério de exclusão quanto a data de publicação no protocolo da revisão sistemática. $\mathrm{O}$ ano em que houve maior número de publicações foi em $2020 \mathrm{com}$ um total de 6 artigos devido ao aumento do uso de laboratórios no período da pandemia.

O primeiro item analisado foi o tipo dos laboratórios descritos nos artigos, podendo ser do tipo remoto ou virtual, conforme já foram descritos na introdução.

Os resultados mostram que a maior parte dos laboratórios implantados é do tipo virtual totalizando 22 laboratórios e 13 são do tipo remoto. Devido à dificuldade de se implantar um laboratório remoto, considerando a necessidade de disponibilizar equipamentos reais para acesso e a dificuldade de executar os experimentos, o número de laboratórios remotos é menor.

Os laboratórios remotos ou virtuais podem ser utilizados tanto para apoio ao ensino presencial, ou seja, servindo como complemento às aulas presenciais quanto para o ensino a distância, aquele em que o aluno não precisa estar fisicamente presente em momento algum 
durante o processo de ensino-aprendizado. Todos os laboratórios verificados foram utilizados para apoio ao ensino presencial nas diversas áreas do conhecimento.

O público-alvo dos laboratórios desenvolvidos é maior para o nível superior [ID 1, 4, 5, $6,7,8,10,11,12,17,20,21,22,28,29,30,33,34$ e 35] em seguida está o nível médio [ID 3, 9, $13,14,15,16,18,23,24,25,26,27,31$ e 32], e poucos laboratórios foram encontrados para o nível fundamental [ID 2 e 19].

Sobre a verificação da aplicabilidade dos laboratórios no ensino, pode-se observar a sua utilização em segmentos diversos, no entanto, a maioria está concentrada na área de Exatas [ID $2,3,4,6,7,8,9,10,11,12,13,15,16,17,18,21,22,23,24,26,27,28,29,31,31,32,33$ e 35], seguido pela área de Biológicas [ID 1, 5, 14, 20, 25 e 34] e por último na área de Humanas [ID 19].

Quanto à descrição das vantagens obtidas com o desenvolvimento do projeto, verificouse que em apenas $24,1 \%$ dos artigos foram evidenciadas vantagens com a implantação dos projetos e estão sumarizadas na Tabela 3. Apenas em dois artigos foram descritas as desvantagens dos projetos e em um deles houve a descrição de alguns problemas técnicos enfrentados na implantação, conforme também apresentado na Tabela 3.

Tabela 3 - Vantagens, desvantagens e problemas identificados nos artigos

\begin{tabular}{|c|c|c|c|}
\hline Tipo & Área & Vantagens Encontradas & ID \\
\hline LV & $\mathrm{E}$ & $\begin{array}{l}\text { Apresenta resultados precisos em certas situações em que é difícil } \\
\text { medir diretamente as grandezas de interesse sem intervenção de } \\
\text { filmagem. }\end{array}$ & [12] \\
\hline LR & $\mathrm{E}$ & $\begin{array}{l}\text { Vantagens verificadas pelos alunos: aprendizado dinâmico, lúdico e } \\
\text { atrativo. }\end{array}$ & [11] \\
\hline LR & E & $\begin{array}{l}\text { Segurança, permitindo que os alunos se concentrem na solução do } \\
\text { problema sem preocupação com acidentes. }\end{array}$ & [22] \\
\hline LR & $\mathrm{E}$ & $\begin{array}{l}\text { Armazenamento de resultados para análise posterior, maior } \\
\text { disponibilidade de acesso ao laboratório. }\end{array}$ & [8] \\
\hline LV & $\mathrm{E}$ & $\begin{array}{l}\text { Possibilidade de repetição do experimento. Realização de } \\
\text { experimentos impossíveis de serem feitos num laboratório real } \\
\text { devido ao alto custo ou alto risco. }\end{array}$ & [7] \\
\hline LV & E & Economia de tempo com instalação e configuração de computadores. & [4] \\
\hline Tipo & Área & Desvantagens Encontradas & ID \\
\hline LR & $\mathrm{B}$ & $\begin{array}{l}\text { Descritas pelos alunos: impossibilidade de corrigir os erros, } \\
\text { dependência de conexão de internet e ter que agendar horário no site. }\end{array}$ & [1] \\
\hline LV & $\mathrm{E}$ & $\begin{array}{l}\text { Aprendizagem complexa para usuários inexperientes em } \\
\text { programação. }\end{array}$ & [7] \\
\hline Tipo & Área & Problemas técnicos descritos & ID \\
\hline LV & $\mathrm{E}$ & $\begin{array}{l}\text { Sobrecarga do servidor por múltiplos acessos. Perda de tempo com o } \\
\text { uso de máquinas virtuais }\end{array}$ & [4] \\
\hline
\end{tabular}

Legenda da Tabela 3: LV - Laboratório Virtual, LR - Laboratório Remoto, E - Exatas, B Biológicas.

Quanto à descrição dos métodos técnicos utilizados no desenvolvimento do laboratório, computaram-se as principais soluções tecnológicas adotadas nos trabalhos analisados, tendo sido descritas em $86,2 \%$ dos artigos. Na Tabela 4 encontram-se as soluções tecnológicas descritas, subdivididas em: Banco de Dados, Ambiente Virtual de Aprendizagem (AVA), Linguagem de Desenvolvimento e Sistemas utilizados.

Tabela 4 - Tecnologias utilizadas nos laboratórios pesquisados

\begin{tabular}{|l|l|}
\hline Banco de dados & MySQL e FireBird \\
\hline Ambiente Virtual de Aprendizagem & Moodle e Sakai \\
\hline Linguagem de desenvolvimento & JAVA, JSP, JSF, VRML, X3D, Blender, AIML \\
\hline
\end{tabular}




\section{Sistemas utilizados}

Ptolemy, OpenSim, Crocodile Chemistry, MIT App

Inventor2, Modellus, Singularity, VISIR e Sloodle

Como pode ser verificado na Tabela 5, os laboratórios encontrados são utilizados em diversas áreas de conhecimento, principalmente os laboratórios do tipo virtual, como Mecânica, Ciência, Fisiologia e Geografia, com uma maior preponderância para a área de Exatas.

Tabela 5 - Laboratórios de ensino encontrados na Revisão Sistemática

\begin{tabular}{|c|c|c|}
\hline \multicolumn{3}{|c|}{ Laboratórios Virtuais } \\
\hline Nome & Quantidade & ID \\
\hline Laboratório de Circuitos Elétricos & 1 & {$[32]$} \\
\hline Laboratório de Física & 9 & {$[3,7,9,13,16,23,26,29,31]$} \\
\hline Laboratório de Fisiologia & 1 & {$[5]$} \\
\hline Laboratório de Geografia & 1 & [19] \\
\hline Laboratório de Hardware & 1 & [4] \\
\hline Laboratório de Hidráulica & 1 & [21] \\
\hline Laboratório de Matemática & 1 & {$[18]$} \\
\hline Laboratório de Química & 3 & {$[20,14,25]$} \\
\hline Laboratório de Redes de Computadores & 2 & {$[10,28]$} \\
\hline Laboratório de Robótica & 1 & {$[27]$} \\
\hline Laboratório de Mecânica & 1 & [12] \\
\hline \multicolumn{3}{|c|}{ Laboratórios Remotos } \\
\hline Laboratório de Automação & 1 & [8] \\
\hline Laboratório de Ciências & 1 & {$[1]$} \\
\hline Laboratório de Eletrônica & 2 & {$[22,33]$} \\
\hline Laboratório de Física & 3 & {$[15,17,24]$} \\
\hline Laboratório de FPGA & 1 & {$[35]$} \\
\hline $\begin{array}{l}\text { Laboratório de Linguagem de } \\
\text { Programação }\end{array}$ & 1 & {$[30]$} \\
\hline Laboratório de Química Experimental & 1 & {$[34]$} \\
\hline Laboratório de Robótica & 3 & {$[2,6,11]$} \\
\hline
\end{tabular}

Constatamos com os dados da Tabela 5 que o maior número de laboratórios descritos nas publicações está relacionado à Física para o ensino médio, sendo 9 laboratórios virtuais e 3 laboratórios remotos para esta disciplina. Este número se justifica nos artigos lidos e seus autores afirmam que a Física é uma matéria que possui tópicos de difícil compreensão [ID 9, 13, 23 e 29] e ainda que "alguns fatos carregam em si a impossibilidade de sua demonstração, devido algumas vezes ao custo, outras vezes ao risco e até mesmo pela raridade do acontecimento" [ID 3]. Além disso, alguns autores também comentam o fato de que as ferramentas de informática facilitam o desenvolvimento de atividades práticas de laboratório e tornam o ambiente de aprendizado mais motivador aos estudantes [ID 16 e 29], e outros mencionam a falta de infraestrutura adequada nas escolas e alto custo de equipamentos como motivação para a implantação de laboratórios [ID 7, 15 e 24].

Foram encontrados dois tipos diferentes de avaliação reportados nos trabalhos analisados. Um tipo de avaliação se refere ao uso do laboratório remoto/virtual e outro tipo de avaliação se refere ao aprendizado do aluno em relação ao conteúdo programático da disciplina. Para efeitos de avaliação do uso do laboratório, por exemplo, o docente ou pesquisador pode enviar questionários aos alunos para saber o que eles acharam do laboratório remoto/virtual utilizado, enquanto para avaliar o aprendizado do aluno, o docente ou pesquisador pode aplicar uma prova com o conteúdo da disciplina para os alunos da turma.

Sendo assim, no âmbito da avaliação de uso, todos os autores descreveram ter obtido feedback dos alunos quanto à usabilidade, ou seja, a experiência dos estudantes quanto ao uso dos 
laboratórios e suas dificuldades, por meio de questionários de avaliação aplicados após a utilização. No entanto, a temática dos questionários tratava apenas sobre a aceitação dos alunos quanto ao laboratório remoto ou virtual além de questões sobre a sua usabilidade.

Por sua vez, no âmbito da avaliação pedagógica sobre o aprendizado dos alunos, apenas em 8 artigos (27,5\%) foram apresentados resultados dos alunos que utilizaram o laboratório como ferramenta de apoio a aprendizagem [ID 7, 10, 18, 21, 24, 26, 28, 29]. Além disso, apenas em $13,8 \%$ dos artigos [ID 7, 18, 21 e 24] houve comparação de resultados entre grupos de alunos que utilizaram o laboratório remoto/virtual em relação a grupos de alunos que utilizaram o laboratório convencional. Nestes artigos foram verificados métodos parecidos para avaliar a aprendizagem do aluno como pode ser observado na Tabela 6 . Uma constatação interessante refere-se à eficácia descrita com o uso do laboratório remoto/virtual. Como pode ser observado na Tabela 6 , dos 8 artigos, 7 chegaram à conclusão de que o uso do laboratório contribuiu com a melhora do aprendizado dos alunos e que o fato pôde ser verificado pelo melhor rendimento dos alunos [ID $7,10,18,21,24,26$ e 28], e apenas em um artigo [ID 29], os resultados obtidos mostraram um rendimento pior.

Tabela 6 - Metodologias utilizadas para verificação da eficácia do sistema

Metodologia Resultados ID

\begin{tabular}{l|l|c}
$\begin{array}{l}\text { Foi realizado o teste com duas } \\
\text { turmas: uma utilizando o } \\
\text { laboratório virtual e outra } \\
\text { utilizando o laboratório } \\
\text { convencional }\end{array}$ & $\begin{array}{l}\text { Verificado maior desempenho na turma que } \\
\text { utilizou o laboratório virtual com relação à turma } \\
\text { que utilizou o laboratório convencional. } \\
\text { A turma que fez uso do laboratório convencional } \\
\text { obteve rendimento melhor. De acordo com o } \\
\text { autor, a turma que utilizou o laboratório virtual } \\
\text { "esperava obter os resultados pela simulação } \\
\text { não encarando como uma ferramenta de auxílio } \\
\text { à aprendizagem". }\end{array}$ & {$[29]$} \\
\hline $\begin{array}{l}\text { Realização de pré-teste e pós- } \\
\text { teste de conhecimentos. }\end{array}$ & $\begin{array}{l}\text { Percebeu-se o aumento da média dos alunos na } \\
\text { avaliação pré e pós-teste. }\end{array}$ & {$[24],[28]$} \\
\hline $\begin{array}{l}\text { Turma com 32 alunos que } \\
\text { utilizaram o laboratório } \\
\text { virtual e elaboram mapa } \\
\text { conceitual sobre os tópicos } \\
\text { aprendidos. }\end{array}$ & $\begin{array}{l}\text { Avaliação do mapa conceitual e aplicação de um } \\
\text { problema de média complexidade em que se } \\
\text { pode observar o aprendizado dos conceitos de } \\
\text { forma significativa. }\end{array}$ & {$[10]$} \\
\hline
\end{tabular}

Ademais, em uma outra análise, foi realizada a contagem dos termos centrais mais utilizados nos artigos por meio do rastreamento das palavras-chave descritas, agrupamento de termos semelhantes e contagem de frequência ou ocorrência desses termos. Para isso, foi utilizado o site https://www.wordclouds.com, tendo sido obtida a nuvem de palavras da Figura 2. Na Figura 2, quanto maior o tamanho da letra utilizada para um determinado termo, maior a sua ocorrência no corpus documental analisado da RSL. 


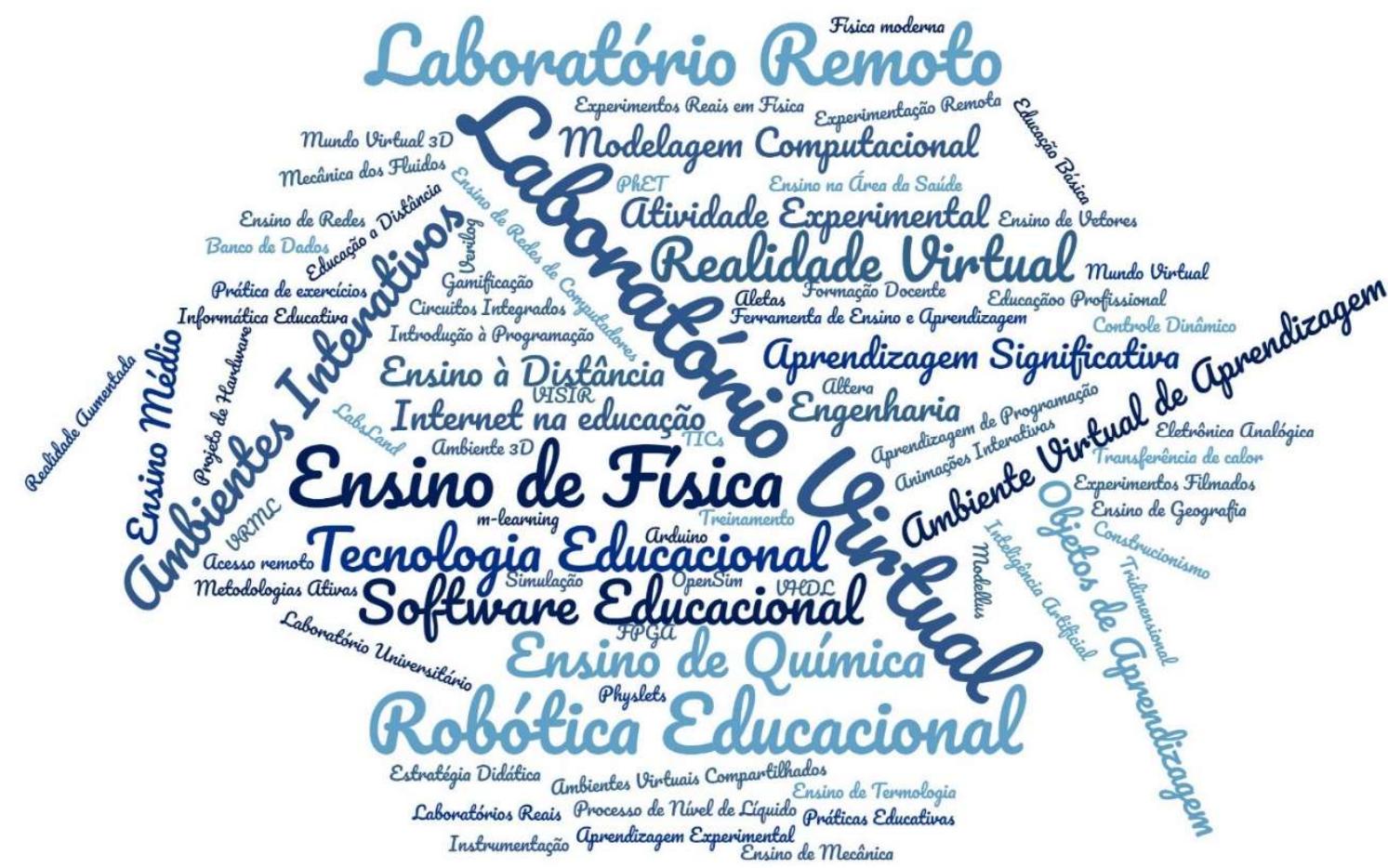

Figura 2 - Nuvem de palavras centrais dos trabalhos encontrados nesta RSL

Dentro deste contexto, vale observar que, embora os laboratórios remotos e virtuais possam ser desenvolvidos para diferentes contextos e práticas profissionais, os principais temas no Brasil têm se centralizado em Ensino de Física, Ensino de Química e Robótica Educacional. Um tema extremamente importante e que tem aparecido apenas de maneira tangencial no corpus documental e por consequente na nuvem de palavras da Figura 2 refere-se às metodologias de ensino e aprendizagem que fundamentam a aplicação de laboratórios remotos e virtuais em contextos educacionais, tendo sido citado pelos autores dos trabalhos analisados as seguintes abordagens educacionais: Aprendizagem Significativa, Metodologias Ativas, Construcionismo e Aprendizagem Experimental.

Como uma análise visando inferir tendências futuras a partir da nuvem de palavras, podese observar que alguns temas atualmente pouco utilizados e analisados nos trabalhos encontrados na RSL provavelmente ganharão mais destaques com o avanço tecnológico: o uso de laboratórios remotos e virtuais em smartphones (termo m-learning da nuvem de palavras); a utilização de mecânicas e da dinâmica de jogos visando o engajamento, a resolução de problemas e o aprimoramento do aprendizado (termo gamificação da nuvem de palavras); a combinação de laboratórios remotos e virtuais em uma simulação computacional que visa imitar a vida ou cotidiano de um estudante em sua instituição de ensino (termos Mundo Virtual 3D e Mundo Virtual da nuvem de palavras); aplicação de inteligência artificial para fins diversificados tendo como base de coleta de dados o histórico, o desempenho e a evolução do estudante em laboratórios remotos e virtuais e; por fim, o uso da realidade virtual e aumentada visando melhorar a experiência e o sensoriamento dos estudantes ao utilizarem os laboratórios remotos e virtuais (termos Realidade Virtual, Realidade Aumentada e Tridimensional da nuvem de palavras).

\section{Conclusões}

Diante das respostas obtidas nesta revisão sistemática, podemos responder à questão de pesquisa (QP1) que, quanto às principais aplicabilidades dos laboratórios remotos e virtuais observamos que têm sido utilizados principalmente para a área de exatas, em cursos de engenharia e, quanto à importância da implantação dos laboratórios remotos e virtuais (QP1), dá-se pelo fato de que os laboratórios remotos ou virtuais complementam o ensino, propiciam ao aluno maior tempo de estudo extraclasse e, também por outros fatores citados em alguns artigos, como por 
exemplo, a falta de recursos para aquisição de equipamentos de alto custo, a falta de pessoal técnico e de espaço físico para implantar laboratórios convencionais. Aqui vale abrir um parêntesis e incluir como um dos fatores de uso dos laboratórios remotos e virtuais a necessidade de mantermos o distanciamento social sem deixar de se realizar as atividades práticas que majoritariamente são realizadas presencialmente em laboratórios especializados. Neste contexto, observa-se que, de acordo com a RSL realizada, houve um aumento extremamente significativo na publicação de artigos em 2020 (ano da não realização de atividades didáticas presenciais nas instituições de ensino devido a pandemia). Para se ter uma ideia dessa dimensão, basta observar que a média de 2004 até 2019 é de aproximadamente 1,81 artigos publicados por ano, enquanto somente em 2020 foram publicados 6 trabalhos sobre o assunto referente ao ensino no Brasil. Em 2018, foram registrados 2 trabalhos e em 2019 nenhum artigo foi registrado na RSL analisada com as bases pesquisadas.

Com relação às estratégias para medir a eficiência do uso de laboratórios remotos/virtuais no processo de aprendizagem dos alunos (QP2), poucos artigos foram encontrados na revisão sistemática que abordaram esse tema. De acordo com a revisão sistemática realizada, em 8 trabalhos pesquisados foi relatada a relação entre o uso do laboratório desenvolvido e a aprendizagem dos alunos, mas em sua maioria, os autores focaram mais em questões técnicas de desenvolvimento do sistema computacional virtual/remoto ou na sua aceitação e usabilidade.

As principais vantagens (QP3) identificadas foram: o aprendizado dinâmico, lúdico, ambiente atrativo, segurança, maior disponibilidade de acesso do laboratório, possibilidade de repetição do experimento e economia de tempo.

As desvantagens (QP3) descritas foram: dependência de conexão de internet, ter que fazer agendamento de horário no site e aprendizagem complexa para usuários inexperientes.

Os problemas técnicos (QP3) apontados foram sobrecarga do servidor por múltiplos acessos e perda de tempo com uso de máquinas virtuais.

Por fim, de acordo com os resultados obtidos na revisão sistemática realizada, que continha 35 artigos, podemos responder à questão principal da seguinte forma: o uso dos laboratórios remotos e virtuais dedicados ao ensino no Brasil ocorre com maior ênfase na área de exatas e com laboratórios virtuais e está presente desde o ensino fundamental até o ensino superior. Pôde-se perceber que muitos estudos estão focados apenas no desenvolvimento e apresentação técnica do projeto e menos em questões pedagógicas decorrentes do uso dos laboratórios virtuais ou remotos deixando de lado um assunto muito importante na área do ensino e educação. "A mera inovação dos recursos tecnológicos não garante a inovação dos processos educacionais" (CALDEIRA, 2004), sendo assim, fazer análise dos impactos e benefícios da estratégia proposta no ambiente de ensino, inclusive comparando com o ensino convencional, traria à instituição de ensino subsídios quanto à implementação de ajustes e melhorias ao projeto, além de trazer ao leitor um panorama mais amplo sobre a proposta educacional. Tópicos interessantes que podem ser endereçados e que foram pouco ou não explorados no corpus documental da RSL analisado envolvem o desenvolvimento de ferramentas para a permitir interação entre estudantes da turma ao utilizarem laboratórios remotos e virtuais, questões sobre a formação docente visando a capacitação tecnológica necessária para aplicação da abordagem em aula, e ainda mais especificamente no contexto nacional, derivação de requisitos, de funcionalidades e implementações que visem contornar problemas da necessidade de infraestrutura e de recursos tecnológicos avançados que não fazem parte de boa parte da população brasileira, visando, com isso, a democratização da tecnologia e um melhor alcance dos laboratórios remotos e virtuais no ensino.

\section{Referências}

ALI, Md Liakot; RAHMAN, Md Habibur; RAHAMAN, Md Abu Nayeem Redwanur. Development of a remote digital system laboratory. In: 2012 15th International Conference on Computer and Information Technology (ICCIT) . IEEE, 2012. p. 575-580.

AMARAL, Érico et al. Laboratório virtual de aprendizagem: uma proposta taxonômica. RENOTE, v. 9, n. 2, 2011. 
BRITO, Lélis Maia de et al. Ambientes virtuais de aprendizagem como ferramentas de apoio em cursos presenciais e a distância. RENOTE, v.11, n.1, 2013.

CALDEIRA, Ana Cristina Muscas. Avaliação da aprendizagem em meios digitais: novos contextos. In: XI Congresso Internacional de Educação a Distância. 2004.

DE FREITAS, Alfredo Américo; BAUCHSPIESS, Adolfo; BORGES, Geovany Araújo. Laboratório de ensino de automação remoto da UnB. In: Anais: Congresso Brasileiro de Ensino de Engenharia. Brasília. 2004.

DA COSTA NETO, Diogo Januario; DA SILVA COSTA, Edmilson Soares. DESAFIOS DO PROFESSOR PRESENCIAL PARA O TRABALHO EM AMBIENTES VIRTUAIS EM ÉPOCAS DE PANDEMIA. Revista Educ@ ção Científica, v. 4, n. 8, p. 1061-1070, 2020.

DE SOUZA, Antonio Lopes; DE OLIVEIRA, José Carlos; DE LIMA SANTOS, Marcelo Paulino. Recursos da computação gráfica para o desenvolvimento de um laboratório virtual de Teoria Eletromagnética. In: Anais do XXIX Congresso Brasileiro de Ensino de Engenharia, Porto Alegre, Setembro de. 2001.

DUARTE MEDINA, Roseclea; TAROUCO, Liane Margarida Rockenbach; AMORETTI, Suzana. Laboratório Virtual ASTERIX-resultados decorrentes da sua utilização como ferramenta cognitiva. In: X Congreso Argentino de Ciencias de la Computación. 2004.

FERENHOF, Helio Aisenberg; FERNANDES, Roberto Fabiano. Desmistificando a revisão de literatura como base para redação científica: método SSF. Revista ACB, v. 21, n. 3, p. 550-563, 2016.

FROZZA, Rejane et al. Agentes pedagógicos emocionais atuando em um ambiente virtual de aprendizagem. RENOTE, v. 9, n. 1, 2011.

GALVÃO, C. M.; SAWADA, N. O.; TREVISAN, M. A. Revisão sistemática: recurso que proporciona a incorporação das evidências na prática da enfermagem. Rev Lat Am Enferm. 2004; 12 (3): 549-56. Rev Esc Enferm USP, v. 43, n. 2, p. 465-71, 2009.

GOMES, Luis et al. Remote experimentation for introductory digital logic course. In: 2009 3rd IEEE International Conference on E-Learning in Industrial Electronics (ICELIE). IEEE, 2009. p. 98-103.

GOMES, Vânia Thais Silva et al. A pandemia da covid-19: repercussões do ensino remoto na formação médica. Revista Brasileira de Educação Médica, v. 44, n. 4, 2020.

GUAITA, Renata Isabelle; GONÇALVES, Fábio Peres. A experimentação na educação à distância: reflexões para a formação de professores de ciências da natureza. In: XI Congresso Brasileiro do Ensino Superior à Distância. 2014. p. 1461-1475.

INDRUSIAK, Leandro Soares; GLESNER, Manfred; REIS, Ricardo. On the evolution of remote laboratories for prototyping digital electronic systems. IEEE Transactions on Industrial Electronics, v. 54, n. 6, p. 3069-3077, 2007.

ZUBIA, J.-G. Programmable Logic and Weblabs. In: Microelectronics Education. Springer, Dordrecht, 2004. p. 277-281.

KITCHENHAM, Barbara; CHARTERS, Stuart. Guidelines for performing systematic literature reviews in software engineering. EBSE Technical Report. 2007. Disponível em: $<$ https://elsevier.com/_data/promis_misc/525444systematicreviewsguide.pdf $>$. Acesso em: 16 de set. 2019.

PERSIANO, Giovanni Vito et al. Distance learning in digital electronics: Laboratory practice on FPGA. In: 2007 IEEE Instrumentation \& Measurement Technology Conference IMTC 2007. IEEE, 2007. p. 1-6.

RAJASEKHAR, Yamuna et al. Teaching FPGA system design via a remote laboratory facility. In: 2008 International Conference on Field Programmable Logic and Applications. IEEE, 2008. p. 687-690. 